\title{
Effects of Sulfate Residues on the Interaction of Basic Fibroblast Growth Factor with Heparin Evaluated by Combination of Computer Modeling and Surface Plasmon Resonance methods
}

\author{
Chao Yapeng ${ }^{1}$, Fan Zheng ${ }^{1}$, Pei Jianfen ${ }^{2 *}$, and Qian Shijun ${ }^{1 *}$ \\ ${ }^{1}$ State Key Laboratories of Transducer Technology, Institute of Microbiology, Chinese Academy of Sciences, \\ Beijing, China 100101; \\ ${ }^{2}$ Center for Theoretical Biology, Academy for advanced interdiciplinatudie, Peking university, Beijing China ,100871 \\ jfpei@pku.edu.cn; qiansj@sun.im.ac.cn
}

\begin{abstract}
Surface plasmon resonance (SPR) technology was utilized for kinetic analysis between basic fibroblast growth factor (bFGF) and heparin derivatives in order to quantitatively investigate crucial sites in the sugar chains. The strongest binary interaction was bFGF/DE-6-OS-heparin $(\mathrm{KD}=1.11 \mathrm{nM})$. Affinity between $\mathrm{N}$-acetyl heparin and bFGF became lower when the amino group was acetylated instead of being sulfated. KD values of bFGF/DE-2-OSheparin, bFGF/DE-OS-heparin decreased dramatically when Osulfates on corresponding sites were removed. This was further confirmed by a molecular modeling based on the computer modeling with SYBYL software. From the electrostatic energy Analysis, the binding energy of $b F G F / h e p a r i n, b F G F / D E-O S-h e p a r i n, ~ b F G F / N-$ acetyl heparin, bFGF/DE-2-OS-heparin, bFGF/DE-6-OS-heparin were $-735,-547,-465,-610,-679$, respectively. DE-OS-heparin. Nacetyl heparin, DE-2-OS-heparin resulted in more loss of binding capability in compared with the original heparin. It was concluded that SPR technology could be used rapidly, quantitatively, sensitively and within real time to detect molecular process of unknown complex sugar chains/growth factor interactions in combination with the computational molecular modeling in the discovering stage from the complex molecular library.
\end{abstract}

Index terms: bFGF; Heparin derivatives; Kinetic Studies; Surface plasmon resonance; Molecular modeling.

\section{Introduction}

Glycosaminoglycans (GAGs) are unbranched polydisperse, acidic polysaccharides, often covalently linked to a protein core to form proteoglycans(PGs). They are characterized (with the exception of keratan sulfate) by a repeating core disaccharide structure comprised of uronic acid and hexosamine residues. The amino group of the hexosamine residue is either $\mathrm{N}$-acetylated or $\mathrm{N}$-sulfated, the uronic acid being either D-glucuronic acid or L-iduronic acid. Moreover, the repeating disaccharide units are $\mathrm{O}$-sulfated to varying degrees at the 3-,4-, or 6-positions of the hexosamine residue and at the 2-position of the uronic acid residues. The most common GAGs are heparin, heparan sulfate (HS), hyaluronic acid (HA), chondroitin sulfate(CS), dermatan sulfate(DS), and keratan sulfate(KS) (Wong, 2003). Heparin/HS are important GAGs with considerable biological significance. Some of their functions were exerted by interaction with growth factors. Structural differences, such as amounts of sulfo groups, have great influence on the ability of heparin/growth factors interactions. Fibroblast growth factors (FGFs) are important heparin binding growth factors, which comprise a large family of developmental and physiological signaling molecules (Ornitz, 2000; Schlessinger et al, 2000).

However, molecular mechanisms such as bFGF/heparin interactions were still unclear though many efforts were made. Various methods have been developed for studying the affinity between biomolecules. Affinity chromatography, ITC, NMR, Fluorescence spectroscopy, size exclusion chromatograohy (SEC), and X-ray technology are extensively used tools. However, they failed to sensitively measure the kinetic constants at the same time (Fuming et al, 2002; Goodger et al, 2008). Surface plasmon resonance (SPR) spectroscopy has become one of established methods for measuring biomolecular interactions. Linhardt's group investigated the interactions between heparin and proteins by immobilizing heparin or proteins on the chips. Moreover, they also proposed a model for the stepwise assembly of a ternary FGF-FGFRheparin complex based on the comparison of SPR sensorgrams (Linhardt and Capila, 2002). Up to data, no systematic kinetic evidence was presented on the interaction between heparin derivatives and bFGF. In this study, kinetic constants for both GAGs and heparin derivatives/bFGF interactions were studied. By comparison of the kinetic data and electrostatic energy, crucial sites in heparin chain that contributed to $\mathrm{bFGF/GAGs}$ interactions were evaluated for the first time.

\section{Materials and Methods}

2.1. Chemicals

Heparin-like polysaccharides and heparin-derived polysaccharides are the following: Heparin (porcine intestinal

This work was supported by National Nature Science Foundation of P.R.China (Project No. 30600118). 
mucosa), Chondroitin sulfate, mescoglycan, dermatan sulfate, $\mathrm{N}$-acetyl heparin, were purchased from Sigma. De-O-Sulfated Heparin , 2-O-Desulfated Heparin , 6-O-Desulfated Heparin were obtained from Neoparin Inc.(America). bFGF (heparin binding growth factor-2, basic brain-derived growth factor, $17 \mathrm{KDa}$ ) was from Invitrogen. Polysialic acid was produced, extracted and refined from the E.coli K12 fermentation. Sensor chip CM5, N-hydroxysuccinimide (NHS), N-ethyl-N(dimethylaminopropyl)-carbodiimide (EDC) and ethanolamine were from BIAcore (Uppasala, Sweden). Bovine serum albumin was from Sigma. Other reagents were either biological or analytical grade.

\subsection{Immobilization of bFGF onto the CM5 sensor chip}

The CM5 sensor chip was activated with a 7-min injection of a 1:1 ratio of $0.4 \mathrm{M}$ EDC (1-ethyl-3-(3-dimethylamino propyl)carbodiimide hydrochloride) and $0.1 \mathrm{M}$ NHS $(N-$ hydroxysuccinimide). The bFGF diluted in HBS-EP buffer (0.01M HEPES, 0.15M NaCl, 3mM EDTA, 0.005\% SP-20, $\mathrm{pH}$ 7.4) was coupled to the surface up to $2000 \mathrm{RU}$. Remaining activated groups were blocked with a 7-min injection of $1 \mathrm{M}$ of ethanolamine ( $\mathrm{pH}$ 8.5). BSA was immobilized on the reference flow cell as a control.

\subsection{Measurements of $b F G F / G A G$ s interactions}

Interactions were characterized using a BIAcore 3000 instrument. Analytes with different concentrations (Heparin porcine intestinal mucosa, Heparan sulfate, Chondroitin sulfate, mescoglycan, dermatan sulfate N-acetyl heparin, polysialic acid ) in HBS-EP were injected over the bFGF chip at a flow rate of $50 \mu \mathrm{l} / \mathrm{min}$. At the end of each sample injection, the HBS-EP buffer was passed over the sensor surface to monitor the dissociation phase. Following $180 \mathrm{~s}$ of dissociation, the sensor surface was fully regenerated by an injection of $10-50 \mu \mathrm{l}$ of $2 \mathrm{M} \mathrm{NaCl}$ in $100 \mathrm{mM}$ sodium acetate buffer(pH 4.0).

\subsection{Analysis of $b F G F / G A G$ s interactions}

Responses were monitored as a function of time (sensorgram) at $25^{\circ} \mathrm{C}$. Reference responses from control flow cells was subtracted from bFGF. Kinetic parameters were evaluated using the BIA Evaluation software version 4.0 (2004). The resulting sensorgrams were used for kinetic parameter determination by globally fitting the experimental data to a 1:1 interaction. Chi square did not exceed $10 \%$ of Rmax for each fit. Association rate constant $\left(k_{\mathrm{a}}\right)$, dissociation rate constant $\left(k_{\mathrm{d}}\right)$, and equilibrium dissociation constant $(K \mathrm{D})$ were calculated using BIAcore evaluation software.

\subsection{Molecular Modeling.}

We also used molecular docking technique to theoretically calculate the binding energies between bFGF and heparin derivatives. The molecular modeling was performed by using SYBYL 7.2 software (SYBYL, version 7.2. St, Louis MO: Tripos Associates; 2006.) installed on a Linux workstation. The three dimensional (3D) structure of bFGF was taken from the Protein Data Bank (http://www.rcsb.org, PDB code $1 \mathrm{BFC})$. 1BFC contains the atomic coordinates of bFGF and its co-crystallized ligand heparin hexasaccharide fragment. We separated the pure bFGF from $1 \mathrm{BFC}$ as the receptor, and heparin hexasaccharide was left as the ligand template. All the models of the heparin derivatives in this study were constructed based on the template by modifying and assembling different fragments, and the modified parts were minimized with Tripos force field with Gasteiger-Hückel charges partial atomic charges. The final molecular conformations were finally minimized again with Tripos force field. All the ligand atoms were assigned with GasteigerHu"ckel charges and all the receptor atoms were assigned with AMBER99 atomic charges. The binding energies between the receptor (bFGF) and the ligands(heparin derivatives) were then calculated with the DOCK procedure implemented in SYBYL 7.2.

\section{Results}

\subsection{Interaction between $b F G F$ and heparin from different organs}

In this study, we measured the association rate constant $\left(k_{\mathrm{a}}\right)$, dissociation rate constant $\left(k_{\mathrm{d}}\right)$, and equilibrium dissociation constant $(K D)$ for binary interactions occurring between bFGF and heparin derivatives (Table 1).

Heparin derivatives such as $\mathrm{N}$-acetyl heparin, DE-2-OSheparin, DE-6-OS-heparin, DE-OS-heparin showed quite different binding activities with bFGF. By comparing with heparin PIM, the affinity between N-acetyl heparin and bFGF was rather lower when amino group was totally acetylated. DE-2-OS-heparin showed even weaker binding activity indicated by $\mathrm{k}_{\mathrm{a}}, \mathrm{k}_{\mathrm{d}}$ and $\mathrm{KD}$ with a decrease of 80.2 fold. While changes of the $\mathrm{k}_{\mathrm{a}}$ and $\mathrm{k}_{\mathrm{d}}$ of DE-6-OS-heparin/bFGF were slight, and binding activity became a little higher than heparin PIM. Fully DE-OS-heparin caused a more than 10-fold decrease of the binding activity though both O-sulfates were substituted. Therefore, the importance of each modification for heparin/bFGF interaction could be indicated as 2-O-sulfate $>$ $\mathrm{N}$-sulfate $>$ total $\mathrm{O}$ - sulfates $>6$-O-sulfate based on the experimental data of KD.

Table 1 Calculated kinetic parameters from the various interaction studies*\#

\begin{tabular}{|l|c|c|c|}
\hline \multicolumn{1}{|c|}{ Interactions } & $\mathrm{k}_{\mathrm{a}}\left(\mathrm{M}^{-1} \mathrm{~s}^{-1}\right)$ & $\mathrm{k}_{\mathrm{d}}\left(\mathrm{s}^{-1}\right)$ & $\mathrm{KD}(\mathrm{M})$ \\
\hline $\mathrm{bFGF} /$ heparin-BL & $2.26 \times 10^{6} \pm 3.42 \times 10^{4}$ & $1.89 \times 10^{-3} \pm 3.47 \times 10^{-5}$ & $8.35 \times 10^{-10}$ \\
\hline $\mathrm{bFGF} /$ heparin-PIM & $2.6 \times 10^{6} \pm 3.25 \times 10^{4}$ & $4.72 \times 10^{-3} \pm 9.98 \times 10^{-5}$ & $1.82 \times 10^{-9}$ \\
\hline $\mathrm{bFGF/N-acetyl} \mathrm{heparin}$ & $1.36 \times 10^{5} \pm 1.63 \times 10^{3}$ & $5.84 \times 10^{-3} \pm 1.33 \times 10^{-4}$ & $4.3 \times 10^{-8}$ \\
\hline $\mathrm{bFGF/} \mathrm{DE-2-OS-heparin}$ & $7.82 \times 10^{4} \pm 7.12 \times 10^{2}$ & $5.24 \times 10^{-3} \pm 1.11 \times 10^{-4}$ & $6.7 \times 10^{-8}$ \\
\hline $\mathrm{bFGF/} \mathrm{DE-6-OS-heparin}$ & $3.2 \times 10^{6} \pm 3.89 \times 10^{4}$ & $3.54 \times 10^{-3} \pm 4.98 \times 10^{-5}$ & $1.11 \times 10^{-9}$ \\
\hline $\mathrm{bFGF/} \mathrm{DE-OS-heparin}$ & $2.35 \times 10^{5} \pm 1.56 \times 10^{3}$ & $4.83 \times 10^{-3} \pm 5.85 \times 10^{-5}$ & $2.06 \times 10^{-8}$ \\
\hline
\end{tabular}

* association rate constant $\left(k_{\mathrm{a}}\right)$, dissociation rate constant $\left(k_{\mathrm{d}}\right)$, and equilibrium dissociation constant $(K D)$ were calculated using BIAcore evaluation software 4.1. Chi square did not exceed $10 \%$ of Rmax for each fit.

\# The data were calculated based on the mean mass of each heparin derivatives since they actual masses were unavailable. 
Other kinds of GAGs or polysaccharide were also applied for the interaction analysis. None of the chondroitin sulfate, dermatan sulfate, mescoglycan and the anion-rich polysaccharide polysialic acid showed obvious interactions with bFGF under the same conditions.

\subsection{Calculated Binding Energy}

To further verify the interaction under each condition, electrostatic energy was calculated by SYBYL 7.2 software using bFGF and basic fragments of the heparin derivatives. The results were shown in Figure 1 and Table 2. Van de waals energy in each case was far lower (below 5 percent in the total energy) thus was not considered here.

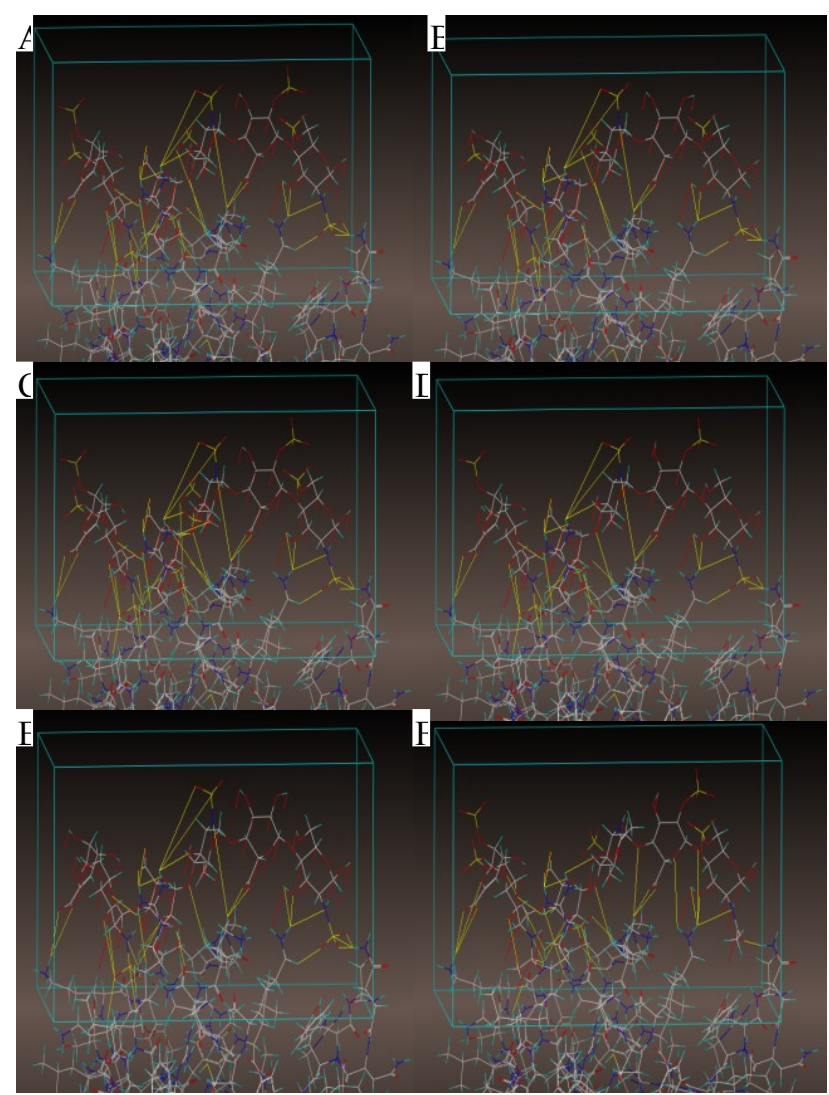

Fig. 1 Electrostatic modeling of bFGF-heparin oligosaccharides interactions. Energy was calculated based on the crystal of bFGF-heparin hexamer complex.

A. Major component in PIM ; B. Heparin-de-2-o- $\mathrm{SO}_{3}{ }^{-}$; C. Major component in BL; D. Heparin-de-6-o- $\mathrm{SO}_{3}{ }^{-}$; E. Heparin-de-2,6-o-SO ${ }_{3}^{-}$; F. Heparin-de-2N-NAc.

In this computer modeling, $\mathrm{N}$-acetyl heparin showed minimal electrostatic energy of -465 during the interaction with bFGF. DE-OS-heparin and DE-2-OS-heparin, followed by -547 and -610 , consistent with the number of sulfates loss in specific sites. DE-6-OS-heparin showed less loss of electrostatic energy suggesting its minor effects in the protein/sugar interaction. Generally, Sulfates were important in the binding capability between the sugar fragments and bFGF, but the structure modification affected the interaction differently in each site. The sequence of binding energy can be summarized as follows: Heparin from PIM $>$ Heparin-DE-6O- $\mathrm{SO}_{3}{ }^{-}>$Heparin-DE-2-O-SO ${ }_{3}{ }^{-}>$Heparin-de- 2,6-O- $\mathrm{SO}_{3}{ }^{-}>$ Heparin-de-2-N-NAc.

Table 2 Calculated electrostatic energy of each heparin derivatives

\begin{tabular}{|l|c|}
\hline \multicolumn{1}{|c|}{ Modification } & Electrostatic energy \\
\hline Heparin from PIM & -735 \\
\hline Heparin from BL & -757 \\
\hline Heparin-de-2-o- $\mathrm{SO}_{3}{ }^{-}$ & -610 \\
\hline Heparin-de-2-N-NAc & -465 \\
\hline Heparin-de-6-o- $\mathrm{SO}_{3}{ }^{-}$ & -679 \\
\hline Heparin-de-2,6-o- $\mathrm{SO}_{3}{ }^{-}$ & -547 \\
\hline
\end{tabular}

\subsection{Fitting analysis between experimental binding affinity and binding energy}

Fitting analysis was conducted based on the experimental data and calculated energy. Minus logarithm of the experimental data was used as abscissa and y-axis. Most of the points fitted well except the bFGF/ DE-2-OS-heparin (Fig. 2). The regression equation was as follows: $y=-155.35 \mathrm{X}+663.06$, with $\mathrm{R}^{2}=0.928$. In SPR analysis, bFGF/DE-2-OS-heparin showed the weakest interaction. But it did not showed the least binding energy accordingly in the molecular modeling.

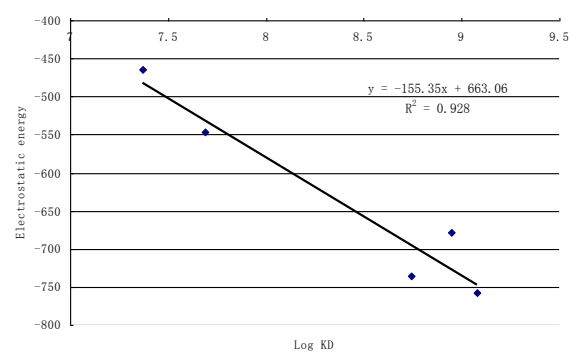

Fig. 2 Relationship between $\lg \mathrm{KD}$ and static energy during the $\mathrm{bFGF} /$ heparin oligosaccharides

\section{Discussion}

As highly sulfated glycosaminoglycan, it is well known that heparin has numerous important biological activities associated with its interaction with diverse proteins. To investigate the molecule basis of its functions, various methods are utilized in studying the binding affinity of heparin with proteins. For most heparin-binding proteins, where there is no such observable change following the binding events, it is difficult to study kinetics of the interaction (Omar et al, 2004). Surface plasmon resonance(SPR), allowing binding events to be sensitively and quantitatively measured in real time, has made it possible to study the kinetics of heparin-protein interactions.

Interaction of FGF (or other growth factors) and heparin was investigate by immobilizing heparin to a streptavidin sensor chip (Fuming et al 2002; Yu et al, 2005). Kinetic data can be obtained using these methods between the growth factors and heparin. But as we know, heparin is a kind of 
multivalent chemical with complex structure, which may result in multiple interactions on the heparin chains. Robinson et al also found that modified heparin oligosaccharides can bind the FGF1 as a 2:1 ratio (Robinson et al, 2005). This study was conducted by immobilization of bFGF2 on the CM5 sensorchip and heparin derivatives were used as the analyte added in solution. Moreover, this method can help to screen and recover affinitive heparin fragments from heparin derived mixture. Due to the complexity of heparin, mean molecular weight of each heparin derivatives was used in the evaluation.

Based on the published crystal structures of FGF·FGFR heparin ternary, two conflicting models have been suggested. The asymmetrical model described a 2:2:1 FGF·FGFR-heparin complex, with the two FGF·FGFR units dimerized in a trans configuration upon a heparin decasaccharide (Pellegrini et al, 2000). In contrast, a symmetrical 2:2:2 FGF·FGFR heparin complex was also proposed (Schlessinger et al, 2000). In this model, the two 1:1:1 complex were held together through direct FGFR1cFGFR1c contacts, as well as secondary FGF2-FGFR1c and heparin-FGFR1c interaction. It was suspected that difference in bioactivity arised though different mechanisms based on the length of the chains and profile of sulfates. Therefore, heparin and its derivatives are known to often bind to target proteins via a two step mechanism. The initial step was crucial since it can not been easily analyzed using X-ray crystallization technology.

As to structural characteristics in heparin chain for specific binding with $\mathrm{bFGF}$, both sites and numbers of sulfates may have contributed. Crystal structures of heparin tetramer/bFGF2 complex, heparin hexamer/bFGF complex have proved that heparin can specifically bind to bFGF by O-sulfates and Nsulfates (Faham et al, 1996). Satoko et al also reported that 2O-DS heparin resulted in dramatic loss of heparin/bFGF binding by immobilizing glycosaminoglycans on a kind of BIAcore SA chip (Ashikari-Hada et al, 2004). In this work, we drew similar conclusion using SPR technology, but disaccord with the conclusion from molecular modeling. Moreover, the contributions of different sites in heparin to the binding with bFGF by constants of various heparin derivatives and bFGF suggested differed from each other. The content of sulfates in heparin $\mathrm{BL}$, with basic disaccharide units of $-\alpha-\delta \mathrm{UA}-2 \mathrm{~S}-$ $[1 \rightarrow 4]$ GlcNS-6S- occupying up to $75-95 \%$, is higher than that of heparin PIM (Guangli et al, 2003). Thus substantial substitution by sulfate group in the heparin backbone did benefit to specific binding with bFGF.

Heparin and other GAGs are known to interact with proteins primarily via ionic interactions between the negatively charged GAGs and positively charged amino acid residues on the surface of proteins. This work investigated it in a combination of SPR technology and molecular modeling, which could make the conclusion more concrete from different aspects. It was found that number of sulfates and $\mathrm{N}$-sulfate in the chains play important roles in the $\mathrm{bFGF} /$ heparin interaction. 2-O-sulfate was suggested to be most crucial by $\mathrm{KD}$ analysis, but the electrostatic energy did not show same effects using molecular model. This was probably caused by the conformational difference between semi-rigid solution structure of heparin and rigid structure of heparin oligosaccharides in the computer modeling (Kahn et al, 2010; Guglieri et al, 2008). 6-O-sulfate was not so important as other sites in the chains.

\section{Conclusions}

1) Extent of sulfation on the heparin backbone -(-(UA[1(4]GlcN- greatly affects the binding constants of heparin/bFGF interaction;

2) Contribution of $\mathrm{O}-, \mathrm{N}$-sulfates to heparin/bFGF binding constants is as follows: 2-O-sulfate $>\mathrm{N}$-sulfate $>$ Total Osulfates $>6$-O-sulfate from the experimental data by SPR technology, but $\mathrm{N}$-sulfate> Total O-sulfates $>2$ 2-Osulfate> >6-O-sulfate from the molecular modeling;

3) Good fitting was obtained between the SPR analysis and molecular modeling except of the bFGF/DE-2O-sulfated heparin interaction.

\section{References}

[1] Ashikari-Hada S., Habuchi H., Kariya Y., Itoh N., Reddi A.H., Kimata K. (2004) Characterization of growth factor-binding structures in heparin/ heparan sulfate using an octasaccharide library. Journal of Biological Chemistry. 279 (13): 12346-54.

[2] Faham S., Hileman R.E., Fromm J.R., Linhardt R.J., Rees D.C. (1996) Heparin structure and interactions with basic fibroblast growth factor. Sciences. 271, 1116-1120.

[3] Fuming Z., Melissa F., Rory M., and Linhardt R. J. (2002) A highly stable covalent conjugated heparin biochip for heparin-protein interaction studies. Analytical Biochemistry. 304, 271-273.

[4] Goodger SJ, Robinson CJ, Murphy KJ, Gasiunas N, Harmer NJ, Blundell TL, Pye DA, Gallagher JT. (2008) Evidence that heparin saccharides promoter FGF2 mitogenesis through two distinct mechanism. Journal of Biological Chemistry. 283 (19): 13001-8.

[5] Guglier S, Hricovíni M, Raman R, Polito L, Torri G, Casu B, Sasisekharan R,Guerrini M. (2008) Minimum FGF2 binding structural requirements of heparin and heparan sulfate oligosaccharides as determined by NMR spectroscopy. Biochemistry. 47(52):13862-9.

[6] Guangli Y., Qun W., Huashi G., Jiamin X., Linhardt R. J. (2003) Preparation of bovine lung heparin oligosaccharides. Journal of ocean university of Qingdao. 32 (2): 231-235.

[7] Linhardt R. J. and Capila I. (2002) Heparin-protein interactions. Angewandte Chemie International Edition. 41, 390-412.

[8] Omar A. I., Fuming Z., C. Sybil L. H., Moosa M., and Linhardt R. J. (2004) Kinetic model for FGF, FGFR, and proteoglycan signal transduction complex assembly. Biochemistry. 43, 4724-4730.

[9] Ornitz D. M. (2000) FGFs, heparan sulfate and FGFRs: complex interactions essential for development . BioEssays. 22: 108-112.

[10] Pellegrini L, Burke DF, von Delft F, Mulloy B, and Blundell TL. (2000) Crystal structure of fibroblast growth factor receptor ectodomain bound to ligand and heparin. Nature 407,1029-1034.

[11] Robinson CJ, Harmer NJ, Goodger SJ, Blundell TL, and Gallagher JT. (2005) Cooperative dimerization of fibroblast growth factor 1 (FGF1) upon a singleheparin saccharide may drive the formation of $2: 2: 1$ FGF1:FGFR2c:heparin trnary complex. Journal of Biological Chemistry. 280 (51): 42274-82Ro.

[12] Schlessinger J., Plotnikov A. N., Ibrahimi O.A., Eliseenkova A.V., Yeh B. K., Yayon A., Linhardt R. J. (2000) Crystal structure of a ternary FGF-FGFR-heparin complex reveals a dual role for heparin in FGF binding and dimerization. Molecular Cell, 6, 743-750.

[13] Tasneem I. And Linhardt R. J. (2003) Chemistry, Biochemistry and Pharma -ceutical potentials of glycosaminoglycans and related saccharides. In: C. H. Wong (ed), Carbohydrate-based drug discovery, Wiley-VCH, Weinheim. 